\title{
Engineering Nano-to-Micron-Patterned Polymer Coatings on Bioresorbable Magnesium for Controlling Human Endothelial Cell Adhesion and Morphology
}

\author{
Wensen Jiang ${ }^{1}$, Chaoxing Zhang ${ }^{1}$, Larry Tran $^{2,3}$, Sebo Gene Wang ${ }^{2,4}$, Ammar Dilshad Hakim², \\ Huinan $\mathrm{Liu}^{1,2 *}$ \\ ${ }^{1}$ Materials Science and Engineering Program, University of California at Riverside. 900 \\ University Avenue, Riverside, CA 92521, United States \\ ${ }^{2}$ Department of Bioengineering, University of California at Riverside. 900 University Avenue, \\ Riverside, CA 92521, United States \\ ${ }^{3}$ Department of Chemical Engineering, University of California at Riverside. 900 University \\ Avenue, Riverside, CA 92521, United States \\ ${ }^{4}$ Department of Chemistry, College of Natural and Agricultural Sciences, University of California \\ at Riverside. 900 University Avenue, Riverside, CA 92521, United States
}

${ }^{*}$ Corresponding Author:

Huinan Liu, Ph.D., Associate Professor Department of Bioengineering Materials Science and Engineering Program University of California at Riverside 900 University Avenue

Riverside, CA 92521

Office: MSE 227

Phone: 9518272944

Fax: 9518276416

Email: huinan.liu@ucr.edu 

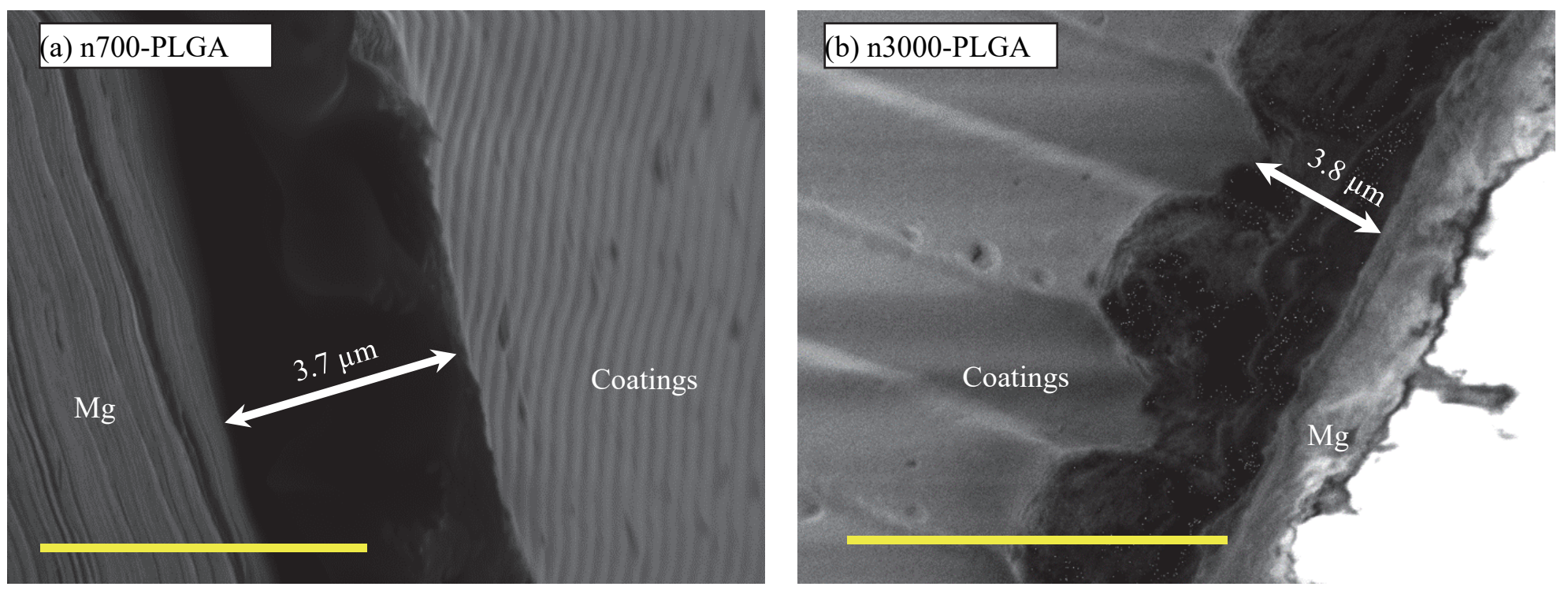

Supplementary Figure S1. Cross-sectional characterization of the nano-to-micron patterned polymer coatings on Mg substrates. (a) Cross-sectional SEM image for n740-PLGA. (b) Cross-sectional SEM image for n3000PLGA. The SEM image in (a) was taken from vertical direction while (b) was taken at $60^{\circ}$ tilt. The thickness of (a) n740-PLGA is $3.7 \mu \mathrm{m}$ and (b) n3000-PLGA is $3.8 \mu \mathrm{m}$. The coatings were cut using a razor blade to expose the cross sections. Scale bar $=5 \mu \mathrm{m}$ for (a) and (b). Original magnification $=8000 \times$ for (a) and (b). 